\title{
Modulation of Morphology, Water Uptake/retention, and Rheological Properties by in- Situ Modification of Bacterial Cellulose With The Addition of Biopolymers
}

PEDDAPAPANNAGARI KALYANI ( $\nabla$ ppgkalyani@iith.ac.in )

IIT Hyderabad https://orcid.org/0000-0002-4799-2209

Mudrika Khandelwal

IITH: Indian Institute of Technology Hyderabad

\section{Research Article}

Keywords: Bacterial cellulose, Bio polymers, Agar, Chitosan, Rheology, surface morphology

Posted Date: March 18th, 2021

DOl: https://doi.org/10.21203/rs.3.rs-295421/v1

License: (c) (1) This work is licensed under a Creative Commons Attribution 4.0 International License. Read Full License 


\section{Abstract}

In situ modification of bacterial cellulose allows structural and morphological tuning which determines the crucial properties such as water absorption/retention and rheological behaviour. This work reports the effect of in situ modification carried out by adding of two biopolymers - Agar and Chitosan - to the standard culture media for bacterial cellulose synthesis. The agar modified BC (Agar-BC) frames the Bacterial cellulose (BC) network as reduced pore volume, and a much denser network, leading to lesser water absorption and further lower retention time than BC. Agar-BC also demonstrates a higher storage modulus, while the yield point is observed at a lower shear strain. This indicates densely packed behaviour of crosslinked polymer with low strain onset of plasticity. On the other hand, chitosan modified $\mathrm{BC}$ (Chitosan-BC) also exhibits a lower pore volume with lower densly packed structure and with lower swellability and water retention reduced to 1 hour (7 hours for BC). Chitosan-BC presents a lower modulus with a yield strain similar to that of unmodified BC. The water absorption-retention behaviour is discussed in details on the basis of relative pore shape-size distribution, fibre dimension and surface area. The mechanism of viscoelatic deformation for each of the cases is explained using a schematic illustrations of the presumed fiber morphologies.

\section{Introduction}

Cellulose is abundant in nature owing to its various sources such as plants, trees, microbes, and algae, which can be processed chemically and enzymatically to obtain cellulose (Lynd et al. 2002). Plant cell walls constitute the major source of cellulose, which also consists of several impurities such as lignin, pectin, hemicelluloses, and xylosans. Thus, intensive and extensive chemical processing is needed to extract pure cellulose for its various applications (Shoda and Sugano 2005; Szymańska-Chargot et al. $2017 b)$. On the other hand, bacterial cellulose (BC) is produced as a $99.99 \%$ pure hydrogel with an ultrafine nanofibrous network. BC possesses good mechanical properties and high water-absorbing and holding capacity (Islam et al. 2017). BC can be easily produced by the fermentation of culture media containing sugars using cellulose producing bacteria (such as acetobacter) in various morphologies such as sheets, spheres under agitated or static conditions (Singhsa et al. 2018). BC and its composites have been found suitable for many applications ranging from merely as an additive in food industries to wound dressings, skin treatments, and filtering membranes (Esa et al. 2014; Lin et al. 2013a). Further, due to its high purity, mechanical strength, crystalline nature, liquid-absorbing, and holding capabilities, biodegradability and most importantly its biocompatibility, BC is of particular interest in drug delivery, bioactive implants, artificial blood vessels, cosmetology and tissue scaffolds (Ullah et al. 2016a).

Due to the wide possibility of its applications, there is a huge interest in the modification of $\mathrm{BC}$ to further tune its properties by adding bio-derived polymers such as Chitosan, Agar, Pectin, Gelatin, Polylactic acid, Polyhydroxybutyrate, Sodium alginate and starch (Bae et al. 2004; Barud et al. 2011; Cacicedo et al. 2020; Kim et al. 2010; Lin et al. 2016; Lin et al. 2013b; Martínez-Sanz et al. 2012; Ruka et al. 2013; SzymańskaChargot et al. 2017a; Wan et al. 2009; Yin et al. 2020; Zhou et al. 2007). It has been observed that the addition of these polymers affects the structure, morphology, and composition which modulates its 
mechanical properties, liquid holding and retention capability, and yield. Such modifications can be carried out by two methods - a) in situ - modifications introduced during cellulose synthesis (Khandelwal et al. 2016) and b) ex-situ modifications - modification introduced after production (Hu et al. 2014; Stumpf et al. 2018). The former is of particular interest as it allows uniform modification and changes at various levels of the hierarchical organization of cellulose. While various studies report in situ modifications and its effect on microstructure, the insights into their impact on crystallinity, pore size distribution, water retention and most importantly on rheological behavior remains poorly explored. Rheological properties are an important consideration in all the above-mentioned applications as they allow understanding of long-term and short term viscoelastic behavior which helps in determining performance, processing, and shelf life.

Amongst the various biopolymers used for modifying the properties of BC, Agar and Chitosan have been chosen for this study, given their high suitability in biomedical, pharmaceutical, and tissue engineering applications. Agar is commonly used in the form of nutrient media for the growth of various bacteria (Basu et al. 2015). Besides its role as a growth medium, it has a pronounced effect on the yield of BC (Bae et al. 2004; Chao et al. 2001; Ishida et al. 2003). Chitosan is a linear polysaccharide produced by the deacetylation of chitin, the second most abundant polysaccharide found on earth (Lim and Hudson 2003; Srinivasa and Tharanathan 2007). Chitosan modified BC finds many applications in food packaging, tissue engineering (Cai et al. 2009; Fernandes et al. 2009; Fu et al. 2009; Kim et al. 2011; Lin et al. 2013b), and in polymeric drug carrier (Cacicedo et al. 2020; Pavaloiu et al. 2014).

While there is abundant literature on ex-situ modifications of $\mathrm{BC}$, the literature on in situ modification by chitosan and agar with a focus on understanding the effect on water absorptivity- retention and rheological properties is absent. This paper looks into the changes in structure, morphology, water absorption, and retention of BC produced by in situ modification with $0.1 \%$ Agar and chitosan, with emphasis on understanding the rheological characteristics.

\section{Materials And Methods 2.1 Materials}

Hestrin Schramm (HS) media was used for the production of BC. The media components include glucose, peptone, yeast extract, citric acid, and disodium hydrogen phosphate of microbiological grade, which were purchased from Himedia, India. Low molecular weight extrapure Chitosan (90\% DA), granulated Agar (bactograde), and Sodium hydroxide (analytical grade) were procured from SRL chemicals, India. Cellulose producing bacteria Komagataeibacter hansenii (ATCC 23769) was procured from LGC Promochem, India.

\subsection{Production of $\mathrm{BC}$ and in situ modification}


Hestrin Schramm (HS) media comprising of glucose $20 \mathrm{~g} / \mathrm{L}$, citric acid $1.15 \mathrm{~g} / \mathrm{L}$, disodium hydrogen phosphate $3.4 \mathrm{~g} / \mathrm{L}$, peptone $5 \mathrm{~g} / \mathrm{L}$ and yeast extract $5 \mathrm{~g} / \mathrm{L}$ was prepared in DI water and adjusted to $\mathrm{pH}$ 4.5 for the production of BC. A $100 \mathrm{~mL}$ of autoclaved media was poured in UV treated petriplates and inoculated with $3 \mathrm{ml}$ of Komagataeibacter hansenii ATCC 23769. The petriplates were wrapped with parafilm sheet and incubated at $26^{\circ} \mathrm{C}$ for 14 days. After 14 days, the produced $\mathrm{BC}$ pellicles were treated with $0.5 \mathrm{M} \mathrm{NaOH}$ to kill the bacteria and then washed with DI water to remove cell debris and neutralize $\mathrm{pH}$. The purified $\mathrm{BC}$ was freeze-dried at $-80^{\circ} \mathrm{C}$ for $24 \mathrm{~h}$. For in situ modification, HS media was modified by adding $0.1 \mathrm{wt} \%$ of Agar and $0.1 \mathrm{wt} \%$ Chitosan to the media for obtaining Agar-BC and Chitosan-BC, respectively. The freeze-dried $B C$ pellicles were used for all characterization studies, except for the rheological measurements where never dried wet pellicles were used. The yield (\%) of BC and modified BC was caluculated from the ratio of weight of dried BC (purified and freeze dried) to the weight of carbon source (D-glucose) used in HS media.

\subsection{Characterization techniques}

The FTIR spectroscopy was performed using an Alpha-P (Bruker Corporation, USA) machine in the wavenumber range of 4000 to $450 \mathrm{~cm}^{-1}$ in transmittance mode to determine the chemical composition. The surface morphology of all freeze-dried samples was observed by a JEOL JSM-7800 Field Emission scanning electron microscope (FE-SEM) operating at an accelerating voltage of $5 \mathrm{kV}$. Before analysis, samples were gently fixed on an aluminum stab with two-sided adhesive tape and gold coated with a sputter coater to deposit 10-15 nm layer of gold. The average fiber diameter was calculated from SEM images with the consideration of at least 100 fibers by using Image $J$ software, across several images. Crystallinity of freeze-dried samples were determined from X-ray Diffraction (XRD) patterns obtained in a

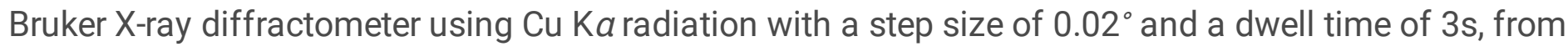
$10^{\circ}$ to $50^{\circ}$ (2 $\theta$ angle). The degree of crystallinity was calculated according to the Equation 2.1.

Crystallinity (\%) $=\frac{\text { Acryst }}{\text { Atotal }} \times 100$

Here, $A_{C r y s t}$ is the sum of area under crystalline peaks; and $A_{\text {Total }}$ is the total area under the diffractogram.

Specific surface area was estimated from Nitrogen adsorption isotherms using an Autosorb iQ, Quantachrome, USA, instrument, using BET analysis. Before analysis, the samples were subjected to degassing at $373 \mathrm{~K}$ for $2 \mathrm{~h}$. Pore size distribution was obtained by $\mathrm{BJH}$ analysis from the isotherm. The porosity of the sample was determined using the following equation Eq.2.2.

Porosity $(\%)=\left[1-\left(\frac{V g}{V a}\right)\right] \times 100$ (Eq. 2.2).

Here, $V_{a}$ is Apparent volume and $V_{g}$ is the actual volume of the sample. 
Mesoporosity (\%) was calculated from the ratio of pore volume for pore size 2-50 $\mathrm{nm}$ to the total pore volume for pore size upto $50 \mathrm{~nm}$.

The water absorption was measured through swelling studies by the gravimetric method (Lin et al. 2013b). The initial weight of the pellicles for a sample size $(2 \mathrm{~cm}$ by $1 \mathrm{~cm})$ was noted after freeze-drying $\left(\mathrm{W}_{1}\right)$. The samples were immersed in a beaker containing $30 \mathrm{ml}$ of deionized water and the wet weight of the swollen membranes $\left(W_{2}\right)$ was measured at various time points. The excess water was removed by gently blotting with a filter paper before weighing. The Water Absorptivity (\%) or swelling was calculated by the following equations:

$W A(\%)=\left(W_{2}-W_{1}\right) / W_{1} * 100$

The water retention of the samples was evaluated as per the reported method (Li et al. 2011). The weighed freeze dried samples $\left(W_{d}\right)$ were completely saturated with water. The saturated samples were wiped with a filter paper to remove excess water and weighed as $W_{0}$. The swollen membranes were placed in a wide-open dish at room temperature. The samples were taken out and weighed $\left(W_{t}\right)$ at several time points and then placed back into the dish. The Water Retention (WR) was calculated as follows.

$\mathrm{WR}(\%)=\left(\mathrm{W}_{\mathrm{t}}-\mathrm{W}_{\mathrm{d}}\right) /\left(\mathrm{W}_{0}\right) * 100 \ldots \ldots \ldots \ldots \ldots \ldots \ldots \ldots$ (Eq 2.4)

These studies were carried out in triplets.

The rheological properties of the samples were measured by using a rheometer (MCR 94 SN82806453, Antonpaar, USA) with parallel plates geometry. Wet pellicles were cut into discs $(25 \mathrm{~mm})$ for amplitude sweep and frequency sweep measurements. Amplitude sweep was used to identify the linear viscoelastic limit to determine the strain for frequency sweep measurement in the scanning range of $0.01-100 \mathrm{~Hz}$ at $26^{\circ} \mathrm{C}$.

\section{Results And Discussion}

\subsection{In situ modified BC production}

The yield for $\mathrm{BC}$, agar-BC, Chitosan-BC was found to be $49 \%, 61 \%$ and $32 \%$, respectively. Agar enhances the yield by increase in viscosity, free cells and regulating the oxygen transfer and also gets incorporated in the pellicle (Bae et al. 2004). On the other hand, the chitosan affects the $\mathrm{pH}$ and interferes with the assembly of cellulose nanofibers reducing the yield.

3.2. Chemical composition determination by FTIR.

The FTIR spectra of BC, Agar-BC, and Chitosan-BC are shown in Figure 1. The common peaks corresponding to cellulose are observed in the spectra corresponsing to $\mathrm{BC}$ - a broad characteristic peak at $3353 \mathrm{~cm}^{-1}$ for $-\mathrm{OH}$ stretching vibration, a peak at $2900 \mathrm{~cm}^{-1}$ attributed to aliphatic $\mathrm{C}-\mathrm{H}$ stretching 
vibration, and a peak at $1436 \mathrm{~cm}^{-1}$ ascribed for $\mathrm{C}-\mathrm{H}$ bending vibrations (Numata et al. 2019; Ullah et al. 2016b). The peaks observed at wavenumbers $1650,1436,1365,1157$ and $1064 \mathrm{~cm}^{-1}$ correspond to glucose carbonyl of cellulose, $-\mathrm{CH}_{2}$ bending vibrations, $-\mathrm{CH}$ bending vibration, $\mathrm{C}-\mathrm{O}-\mathrm{C}$ asymmetric stretching of glycoside bond and C-O stretching, respectively (Kačuráková et al. 2002). Therefore, The FTIR spectra confirm that the production of pure cellulose.

The FTIR absorption spectra of Agar-BC is similar to pure BC spectra, except that the intensity of peaks for - $\mathrm{OH}, \mathrm{CH}_{2}, \mathrm{C}-\mathrm{H}, \mathrm{C}-\mathrm{O}-\mathrm{C}$ increased as agar comprises of galactose monomer units, which has same functional groups as cellulose. The peaks at 1157, 1114, and $1064 \mathrm{~cm}^{-1}$ indicate the presence of $\mathrm{C}_{2} \mathrm{O}_{2}$, $\mathrm{C}_{3} \mathrm{O}_{3}$, and $\mathrm{C}_{6} \mathrm{O}_{6}$ respectively (Shah et al. 2010). Thus, the FTIR spectra confirm that Agar is incorporated into the $\mathrm{BC}$.

The Chitosan-BC spectrum was also similar to that of pure $B C$ because most functional groups are common to both cellulose and chitosan (Munim et al. 2020; Urbina et al. 2018). The -OH stretching peak at $3358 \mathrm{~cm}^{-1}, \mathrm{CH}$ stretching at $2896 \mathrm{~cm}^{-1}$ and bending vibration peak at $1450 \mathrm{~cm}^{-1}$ for $\mathrm{a}-\mathrm{CH}_{2}$ bending are observed and an extra peak for $\mathrm{C}=0$ stretching is seen at $1737 \mathrm{~cm}^{-1}$, which is mainly a characteristic of acetyl groups (Wong and Chan 2018). There is a peak shift to lower wave numbers for amide bond, which can be ascribed to its involvement in hydrogen bonding. (Munim et al. 2020).

\subsection{Morphology and microstructure determination by SEM}

The microstructures of $B C$, Agar-BC and Chitosan-BC were illustrated in Figure 2 ( $a, b$, and $c)$, which reveals a typical interwoven nanofibrous morphology. The average fibril diameter of $B C$ was found to be $58 \mathrm{~nm} \pm 15 \mathrm{~nm}$, which is similar to the earlier reports (Khandelwal et al. 2016; Xiang and Acevedo 2017). The fibers of Agar-BC were more aggregated and produced a denser network than BC. The average fiber diameter of Agar-BC was $80 \mathrm{~nm} \pm 25 \mathrm{~nm}$ which is higher and more widely distributed than that of $\mathrm{BC}$. On the other hand, the average fibril diameter of Chitosan-BC was found to be lower than $B C$ with a value of $49 \mathrm{~nm} \pm 19 \mathrm{~nm}$. Another interesting observation from the SEM images is that the Agar-BC nanofibers appear to be more bundled and coiled-up as compared to the fibres present in Chitosan-BC, where the fibers are straighter and more distinct. This has important implication on pore size and shape which determines the swellability and rheology.

\subsection{Structural analysis by XRD}

The X-ray diffraction profiles of BC, Agar-BC and Chitosan-BC are shown in Fig. 3 and the crystallinity values are tabulated in Table 1. The diffraction peaks of pure BC was obtained at 2 theta angle of $14.6^{\circ}$, $16.7^{\circ}$ and $22.7^{\circ}$ which correspond to the primary diffraction peak from crystal planes of (100), (010), and (110) in la cellulose. (Fang and Catchmark 2015; Kim et al. 2010; Mohite and Patil 2014). The crystallinity of pure $\mathrm{BC}$ was found to be $77 \%$. Agar-BC shows similar crystalline diffraction pattern as BC with distinct 
peaks at $2 \theta=14.6^{\circ}, 16.7^{\circ}, 22.7^{\circ}$ and $34.1^{\circ}$ and a higher crystallinity of $83 \%$. It may be envisaged that the addition of agar to media increases the viscosity of the media which can reduce the disorder in the assembly of cellulose chains and thus can improve the crystallinity of BC. The diffraction pattern for Chitosan-BC was also similar to that of $B C$ where the 3 major peaks at 2 theta angles of $14.6^{\circ}, 16.7^{\circ}$ and $22.7^{\circ}$ are seen and the crystallinity was found to be $75 \%$. The decrease in crystallinity can be attributed to the interference of chitosan molecules in the cellulose chain assembly process. (Urbina et al. 2018; Zhijiang et al. 2011).

\subsection{Surface area, and pore size distribution by $\mathrm{N}_{2}$ adsorption}

The specific surface area of $\mathrm{BC}$, Agar-BC and Chitosan-BC was measured by $\mathrm{N}_{2}$ adsorption isotherms shown in Figure 4 (a) and the estimated pore size distribution is depicted in Figure 4 (b). The isotherms of all the samples are type IV isotherm with a hysteresis loop. Hysteresis at mid to high relative pressures is attributed to capillary condensation in mesopores. Particularly here, a H3 type of hysteresis is seen which suggests slit-like pores. In case of Chitosan-BC, low pressure hysteresis is also seen which can be attributed to the swelling of non-rigid slit like pores with infinite pore length, which may originate from lower interactions between the chitosan modified cellulose fibers and low density.

The BET specific surface area (Table 1) of BC was found to be $74.38 \mathrm{~m}^{2} / \mathrm{g}$ while that for Agar-BC and Chitosan-BC was $53.37 \mathrm{~m}^{2} / \mathrm{g}$ and $25.98 \mathrm{~m}^{2} / \mathrm{g}$ respectively. The pore volume of $\mathrm{BC}$, Agar-BC and Chitosan$B C$ were found to be $0.245 \mathrm{~cm}^{3} / \mathrm{g}, 0.176 \mathrm{~cm}^{3} / \mathrm{g}$, and $0.174 \mathrm{~cm}^{3} / \mathrm{g}$ respectively. Overall porosity of $\mathrm{BC}$, Agar-BC and Chitosan-BC was found to be $88.2 \%, 86.1 \%$ and $87.8 \%$. The calculated relative mesoporosity with respective to microporosity of BC, Agar-BC and Chitosan-BC was found to be $74 \%, 93 \%$ and $82 \%$. The relative microporosity of modified $\mathrm{BC}$ was reduced or in other words the relative mesoporosity was increased using modification, the reason for which needs further investigation. However, it may be attributed to filling up of the micropores in case of Agar, while in case of chitosan, it is difficult to comment as it comprises of slit like pores. In case of Agar-BC, it can be clearly seen that the fiber diameter has increased which can contribute to lesser surface area while in the case of Chitosan-BC, relatively larger number of larger pores can cause an overall reduction in surface area. However, it must be remembered that in case of hysteresis at low pressure, BET surface area may not be accurate.

\subsection{Water absorptivity and retention kinetics of BC Modifiers}

The water absorption behavior of BC modifiers and pure BC is presented in Figure.5 (a) and also summarized in Table 1. The Water absorptive kinetics showed that after $6 \mathrm{hrs}$, the samples were almost saturated when immersed in deionized water. All the curves show three phases - a) a steep slope in the first 1 hour reaching up to about $95 \%, 75 \%$ and $65 \%$, for BC, Agar-BC and Chitosan-BC, respectively followed by b) slowing up of uptake and c) a saturation plateau at 150\% (achieved in 6 hours), 130\% ( achieved in 5 hours) and 110\% (achieved in 5 hours), for BC, Agar-BC and Chitosan-BC, respectively. The slowing down followed by increasing uptake may be because of further ingress of water into the interiors. 
The water absorptivity rate and extent of absorption for Agar-BC is lesser than $\mathrm{BC}$ due to a) denser network evident from SEM images, b) lower surface area and pore volume revealed by Nitrogen adsorption studies and c) lesser tendency to rearrange, given the higher crystallinity of nanofibers. The water absorptivity of Chitosan-BC was also lesser than BC due to lesser surface area and less dense network.

The water retention behavior for the samples is shown in Figure 5 (b), where distinct regions with a fast removal of unbound water, followed by sustained weight-loss, and a very slow evaporation rate in the final step, can be observed. BC showed a higher water retention with more retention time of 7 hours due to higher water content and a deep ultra-fine network with comparatively higher microporosity. The water retention by Agar-BC and Chitosan-BC was seen up to 6 hours and 1 hour respectively, owing to lower water content and lesser microporosity. The structural and morphological variations in $\mathrm{BC}$ greatly influences the water uptake-retention kinetics.

Table 1. Results obtained from XRD, BET, Water Absorptivity and Water Retention Kinetics

\begin{tabular}{|llllll|}
\hline $\begin{array}{l}\text { Sample } \\
\text { name }\end{array}$ & $\begin{array}{l}\text { Crystallinity } \\
(\%)\end{array}$ & $\begin{array}{l}\text { Surface area } \\
\left(\mathrm{m}^{2} / \mathrm{g}\right)\end{array}$ & $\begin{array}{l}\text { Pore } \\
\text { volume } \\
\left(\mathrm{cm}^{3} / \mathrm{g}\right)\end{array}$ & $\begin{array}{l}\text { Maximum } \\
\text { WAC } \\
(\%)\end{array}$ & $\begin{array}{l}\text { Maximum } \\
\text { WRT } \\
(\mathrm{h})\end{array}$ \\
\hline BC & 77 & 74.38 & 0.245 & 153 & 7.0 \\
\hline Agar-BC & 83 & 53.37 & 0.176 & 130 & 6.0 \\
\hline Chitosan-BC & 75 & 25.98 & 0.174 & 110 & 1.0 \\
\hline
\end{tabular}

\subsection{Rheological behavior}

The dynamic viscoelastic parameters, storage modulus $\left(G^{\prime}\right)$ and loss modulus $\left(G^{\prime \prime}\right)$, of BC and modified $B C$ are shown as a function of shear strain in Figure 6. In linear viscoelastic region (LVER), G' values are higher than that of G", which is a signature of gel-like solid behavior. The G' values are constant at low strains and later decreases with strain. The point of deviation from linearity is referred to as the yield point (limit of the LVER), where irreversible structural deformation such as junction disruptions start happening. The calculated yield stresses for all the samples is tabulated in Table 2. It can be seen that the yield stress values are highest for Agar-BC and lowest for Chitosan-BC.

Further, the flow point is defined as the point of intersection of storage and loss modulus curves $\left(G^{\prime}=G^{\prime \prime}\right)$ when the material shows a change in behavior from solid gel-like to a viscous liquid-like property. The strain and stress corresponding to the flow point are also tabulated in Table 2. It is interesting to observe 
that while the flow stress values are highest for Agar-BC, the flow strain values are highest for $\mathrm{BC}$. Chitosan-BC demonstrates the lowest flow stress as well as strain.

Overall, a higher storage modulus values with smaller values of yield and flow strains are observed for Agar-BC, as compared to $\mathrm{BC}$. This implies Agar-BC possesses a more gel strength or rigid network requiring a higher shear stress for deformation. However, the flow stress values of both, $B C$ and Agar-BC, are comparable as in one case strain is higher while in the other case modulus is higher. This implies that $\mathrm{BC}$ is a more reliable hydrogel which retains its elastic nature for larger strain, and for low strain, Agar-BC offers a higher modulus, making it more suitable .

Further, the Chitosan-BC is lower in modulus and also flows at lower strain, but yields at strain higher than Agar-BC and similar to $\mathrm{BC}$. In other words, Chitosan-BC allows perhaps higher elastic strain before permanent changes. However, owing to lesser interconnections evident in the SEM images, the flow point is observed at lower strain and stress. This makes Chitosan-BC a more pliable and processible hydrogel. Broadly it can be said that Chitosan-BC behaves more like a low modulus plastic material (low modulus, low strain transition), while Agar-BC behaved like a high modulus plastic material (high modulus, low strains transition) with Agar acting as a reinforcement polymer to BC.

Table 2: Viscoelastic parameters for BC, Agar-BC and Chitosan-BC

\begin{tabular}{|llllll|}
\hline Sample & $\begin{array}{l}\text { Yield stress }\left(\mathrm{G}^{\prime}\right) \\
{[\mathrm{kPa}]}\end{array}$ & $\begin{array}{l}\text { Yield } \\
\text { point } \\
\mathrm{Y}(\%)\end{array}$ & $\begin{array}{l}\text { Flow point modulus }\left(\mathrm{G}^{\prime}=\right. \\
\left.\mathrm{G}^{\prime \prime}\right)[\mathrm{kPa}]\end{array}$ & $\begin{array}{l}\text { Flow } \\
\text { point } \\
\left(\mathrm{G}^{\prime}=\mathrm{G}^{\prime \prime}\right)\end{array}$ & $\begin{array}{l}\text { Flow stress } \\
{[\mathrm{kPa}]}\end{array}$ \\
\hline $\mathrm{BC}$ & 116 & & $\mathrm{Y}(\%)$ & \\
\hline Agar-BC & 170 & 0.0485 & 4.8 & 23.32 & 1.564 \\
\hline $\begin{array}{l}\text { Chitosan- } \\
\text { BC }\end{array}$ & 16 & 0.019 & 11.8 & 10.02 & 1.677 \\
\hline
\end{tabular}

Further, an angular frequency sweep was carried out at strains within the LVER limits for each sample. The storage modulus $\left(G^{\prime}\right)$, loss modulus $\left(G^{\prime \prime}\right)$ and complex viscosity $\left(\eta^{\star}\right)$ of all samples are plotted as a function of angular frequency in Figure $7(\mathrm{a} \& \mathrm{~b})$. The higher values of storage modulus $(G)$ as compared to loss modulus $\left(G^{\prime \prime}\right)$ in the entire frequency range confirms the retention of gel-like solid behavior for short as well as long relaxation times. This makes these materials stable and thus symbolizes a good shelf life. It is also seen that both moduli were independent of frequency in the all hydrogels. The complex viscosity of all samples decreases linearly with an increase in frequency indicating a shearthinning behavior.

For further understanding, the deformation behavior of $\mathrm{BC}$ hydrogel can be understood using the morphological illustration for interaction of fibers network among the BC modifiers, as shown in Figure 8. 
This illustration can explain the deformation behavior of bacterial cellulose, Agar-BC and Chitosan-BC as a application of shear stress. The initial strains would lead to an elastic deformation followed by a combination of elastic and viscous deformation at higher strains. In case of Agar-BC, depicting the higher gel strength nature which shows plastic deformation at low strains. This would be a specific hygrogen bonding between $\mathrm{BC}$ fibers and agar. So that which strengthens the $\mathrm{BC}$ network. In case of Chitosan-BC, there might be a non specific bonding like eclectrostatic interactions between $\mathrm{BC}$ fibers and amine charge molecules of Chitosan. So that it might loosen the strength of BC network

\section{Conclusion}

The possibility of modulating water uptake-retention and rheological behaviour by tuning the morphology and structure has been successfully demonstrated by using In situ modification of bacterial cellulose. With the addition of Agar and chitosan leads to considerable changes in fibre dimensions, pore sizeshape and surface area. Agar-BC exhibited a lower water uptake owing to denser and rigid network, while Chitosan-BC showed a huge reduction in water uptake as well as retention time, due to lower densly packed network. The modifications also led to an overall reduction in micropores (as compared to mesopores). The rheological properties reveal higher gel strength of agar-BC and a lower gel strength of Chitosan-BC. Which is due to interaction of biopolymers with pure cellulose makes various woven network of BC. Agar acts as a reinforcement at very low strains leading to increase in strength, which eventually deforms and lead to flow at relatively lower strains. Chitosan is known to interfere with cellulose assembly during synthesis leading to lower yield and thus a weaker network. The rheological measurements also establish the logn term stability of materials within the elastic limit. This work successfully demonstrates the possibility to tune morphological, structural, water absorption- retention and rheological behavior of BC by in situ modification in terms of crystallinity, fiber network, surface area, pore size distribution, water swelling-sustain kinetics, yield stress and flow stress, which are essential parameters to determine stability and surface morphology as application of drug carriers and regenerative medicine and also in the processing of food and cosmetics due to its extensive biodegradable nature of these bio polymers.

\section{Abbreviations}

BC- Bacterial Cellulose; HS- Hestrin Schramm; DI- Deionized; DA- Deacetylation; Agar-BC- Agar modified Bacterial Cellulose; Chitosan-BC- Chitosan modified Bacterial Cellulose; BET- Brunauer-Emmett-Teller; BJH- Barrett-Joyner-Halenda; WA- Water Absorptivity; WR- Water Retention; LVER- Linear Viscoelastic Region.

\section{Declarations}




\section{Acknowledgment}

We thank Pankaj Choubey and Tithi Basu from IIT Hyderabad for assisting in rheological measurements. We also acknowledge financial support from AT\&T CSR funds and SERB, India.

\section{Conflict of interest}

We declare no conflict of interest.

\section{References}

1. Bae S, Sugano Y, Shoda M (2004) Improvement of bacterial cellulose production by addition of agar in a jar fermentor. Journal of bioscience bioengineering 97:33-38

2. Barud HS, Souza JL, Santos DB, Crespi MS, Ribeiro CA, Messaddeq Y, Ribeiro SJ (2011) Bacterial cellulose/poly (3-hydroxybutyrate) composite membranes. Carbohyd Polym 83:1279-1284

3. Basu S, Bose C, Ojha N, Das N, Das J, Pal M, Khurana S (2015) Evolution of bacterial and fungal growth media Bioinformation 11:182

4. Cacicedo ML, Pacheco G, Islan GA, Alvarez VA, Barud HS, Castro GR (2020) Chitosan-bacterial cellulose patch of ciprofloxacin for wound dressing: Preparation and characterization studies International. Journal of Biological Macromolecules 147:1136-1145

5. Cai Z, Jin H-J, Kim J Chitosan blended bacterial cellulose as a smart material for biomedical application. In: Nanosensors, biosensors, and info-tech sensors and systems 2009, 2009. International Society for Optics and Photonics, p 72910 U

6. Chao Y, Mitarai M, Sugano Y, Shoda M (2001) Effect of addition of water-soluble polysaccharides on bacterial cellulose production in a 50-L. airlift reactor Biotechnology progress 17:781-785

7. Esa F, Tasirin SM, Rahman NA (2014) Overview of bacterial cellulose production and application Agriculture and Agricultural Science Procedia 2:113-119

8. Fang L, Catchmark JM (2015) Characterization of cellulose and other exopolysaccharides produced from Gluconacetobacter. strains Carbohydrate polymers 115:663-669

9. Fernandes SC, Oliveira L, Freire CS, Silvestre AJ, Neto CP, Gandini A, Desbriéres J (2009) Novel transparent nanocomposite films based on chitosan and bacterial cellulose. Green Chem 11:20232029

10. Fu LN, Wang W, Yu LJ, Zhang SM, Yang G Fabrication of novel cellulose/chitosan artificial skin composite. In: Materials Science Forum (2009) Trans Tech Publ, pp 1034-1038

11. Hu W, Chen S, Yang J, Li Z, Wang H (2014) Functionalized bacterial cellulose derivatives and nanocomposites. Carbohydrate polymers 101:1043-1060

12. Ishida T, Mitarai M, Sugano Y, Shoda M (2003) Role of water-soluble polysaccharides in bacterial cellulose production. Biotechnol Bioeng 83:474-478 
13. Islam MU, Ullah MW, Khan S, Shah N, Park JK (2017) Strategies for cost-effective and enhanced production of bacterial cellulose. Int J Biol Macromol 102:1166-1173

14. Kačuráková M, Smith AC, Gidley MJ, Wilson RH (2002) Molecular interactions in bacterial cellulose composites studied by 1D FT-IR and dynamic 2D FT-IR. spectroscopy Carbohydrate Research 337:1145-1153

15. Khandelwal M, Windle AH, Hessler N (2016) In situ tunability of bacteria produced cellulose by additives in the culture media. Journal of Materials Science 51:4839-4844. doi:10.1007/s10853016-9783-0

16. Kim J, Cai Z, Chen Y (2010) Biocompatible bacterial cellulose composites for biomedical application Journal of nanotechnology in Engineering and Medicine 1

17. Kim J, Cai Z, Lee HS, Choi GS, Lee DH, Jo C (2011) Preparation and characterization of a bacterial cellulose/chitosan composite for potential biomedical application. J Polym Res 18:739-744

18. Li H, Yang J, Hu X, Liang J, Fan Y, Zhang X (2011) Superabsorbent polysaccharide hydrogels based on pullulan derivate as antibacterial release wound dressing. Journal of Biomedical Materials Research Part A 98:31-39

19. Lim S-H, Hudson SM (2003) Review of chitosan and its derivatives as antimicrobial agents and their uses as textile chemicals Journal of macromolecular science, part C. Polymer reviews 43:223-269

20. Lin S-P, Calvar IL, Catchmark JM, Liu J-R, Demirci A, Cheng K-C (2013a) Biosynthesis production applications of bacterial cellulose Cellulose 20:2191-2219

21. Lin S-P, Liu C-T, Hsu K-D, Hung Y-T, Shih T-Y, Cheng K-C (2016) Production of bacterial cellulose with various additives in a PCS rotating disk bioreactor and its material. property analysis Cellulose 23:367-377

22. Lin W-C, Lien C-C, Yeh H-J, Yu C-M, Hsu S-h (2013b) Bacterial cellulose and bacterial cellulosechitosan membranes for wound dressing applications. Carbohydrate polymers 94:603-611

23. Lynd LR, Weimer PJ, van Zyl WH, Pretorius IS (2002) Microbial cellulose utilization: fundamentals and biotechnology Microbiol. Mol Biol Rev 66:506-577. doi:10.1128/mmbr.66.3.506-577.2002 table of contents

24. Martínez-Sanz M, Lopez-Rubio A, Lagaron JM (2012) Optimization of the dispersion of unmodified bacterial cellulose nanowhiskers into polylactide via melt compounding to significantly enhance barrier and mechanical properties Biomacromolecules 13:3887-3899

25. Mohite BV, Patil SV (2014) Physical, structural, mechanical and thermal characterization of bacterial cellulose by G. hansenii NCIM 2529. Carbohydrate polymers 106:132-141

26. Munim SA, Saddique MT, Raza ZA, Majeed MI (2020) Fabrication of cellulose-mediated chitosan adsorbent beads and their surface chemical characterization. Polym Bull 77:183-196

27. Numata Y, Kono H, Mori A, Kishimoto R, Tajima K (2019) Structural and rheological characterization of bacterial cellulose gels obtained from. Gluconacetobacter genus Food hydrocolloids 92:233-239 
28. Pavaloiu R-D, Stoica-Guzun A, Stroescu M, Jinga SI, Dobre T (2014) Composite films of poly (vinyl alcohol)-chitosan-bacterial cellulose for drug controlled release. Int J Biol Macromol 68:117-124

29. Ruka DR, Simon GP, Dean KM (2013) In situ modifications to bacterial cellulose with the water insoluble polymer poly-3-hydroxybutyrate. Carbohydrate polymers 92:1717-1723

30. Shah N, Ha JH, Park JK (2010) Effect of reactor surface on production of bacterial cellulose and water soluble oligosaccharides by Gluconacetobacter hansenii. PJK Biotechnology Bioprocess Engineering 15:110-118

31. Shoda M, Sugano Y (2005) Recent advances in bacterial cellulose production. Biotechnol Bioprocess Eng 10:1

32. Singhsa P, Narain R, Manuspiya H (2018) Physical structure variations of bacterial cellulose produced by different Komagataeibacter xylinus strains and carbon sources in static and agitated conditions. Cellulose 25:1571-1581

33. Srinivasa P, Tharanathan R (2007) Chitin/chitosan-Safe, ecofriendly packaging materials with multiple potential uses. Food reviews international 23:53-72

34. Stumpf TR, Yang X, Zhang J, Cao X (2018) In situ and ex situ modifications of bacterial cellulose for applications in tissue engineering. Materials Science Engineering: C 82:372-383

35. Szymańska-Chargot M, Chylińska M, Cybulska J, Kozioł A, Pieczywek PM, Zdunek A (2017a) Simultaneous influence of pectin and xyloglucan on structure and mechanical properties of bacterial cellulose composites. Carbohydrate polymers 174:970-979

36. Szymańska-Chargot M, Chylińska M, Gdula K, Kozioł A, Zdunek A (2017b) Isolation and characterization of cellulose from different fruit and vegetable pomaces Polymers 9:495

37. Ullah H, Santos HA, Khan T (2016a) Applications of bacterial cellulose in food. cosmetics drug delivery Cellulose 23:2291-2314

38. Ullah MW, Ul-Islam M, Khan S, Kim Y, Park JK (2016b) Structural and physico-mechanical characterization of bio-cellulose produced by a cell-free system. Carbohydrate polymers 136:908916

39. Urbina L, Guaresti O, Requies J, Gabilondo N, Eceiza A, Corcuera MA, Retegi A (2018) Design of reusable novel membranes based on bacterial cellulose and chitosan for the filtration of copper in wastewaters Carbohydrate polymers 193:362-372

40. Wan Y, Luo H, He F, Liang H, Huang Y, Li X (2009) Mechanical, moisture absorption, and biodegradation behaviours of bacterial cellulose fibre-reinforced starch biocomposites. Composites Science Technology 69:1212-1217

41. Wong JYM, Chan MY (2018) Influence of bleaching treatment by hydrogen peroxide on chitosan/durian husk cellulose biocomposite films Advances. in Polymer Technology 37:2462-2469

42. Xiang C, Acevedo NC (2017) In situ self-assembled nanocomposites from bacterial cellulose reinforced with eletrospun poly (lactic acid)/. lipids nanofibers Polymers 9:179 
43. Yin N, Du R, Zhao F, Han Y, Zhou Z (2020) Characterization of antibacterial bacterial cellulose composite membranes modified with chitosan or. chitooligosaccharide Carbohydrate Polymers 229:115520

44. Zhijiang C, Chengwei H, Guang Y (2011) Retracted: Preparation and characterization of a bacterial cellulose/chitosan composite for potential biomedical application. J Appl Polym Sci 121:1488-1494

45. Zhou L, Sun D, Hu L, Li Y, Yang J (2007) Effect of addition of sodium alginate on bacterial cellulose production by Acetobacter xylinum. J Ind Microbiol Biotechnol 34:483

Figures

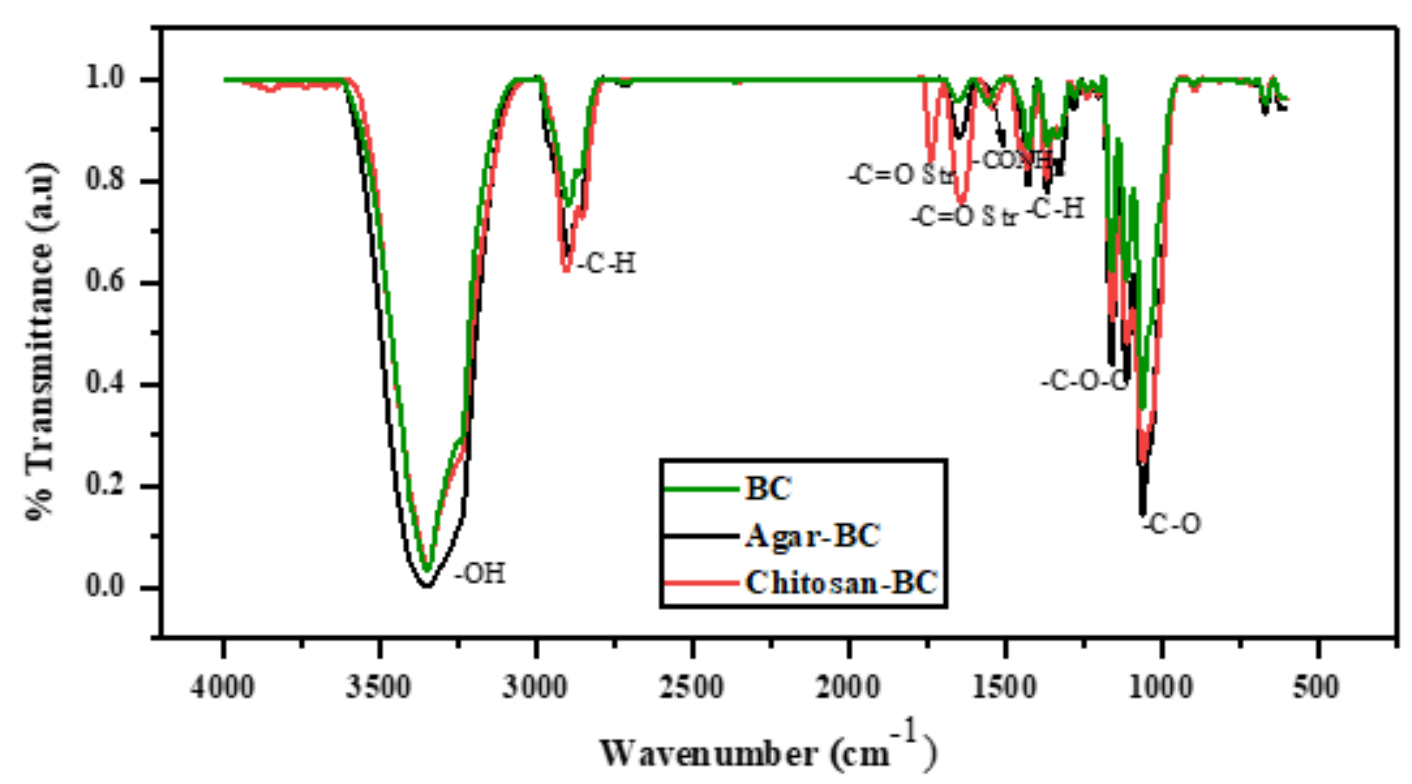

Figure 1

FTIR Spectra of BC, Agar-BC and Chitosan-BC 

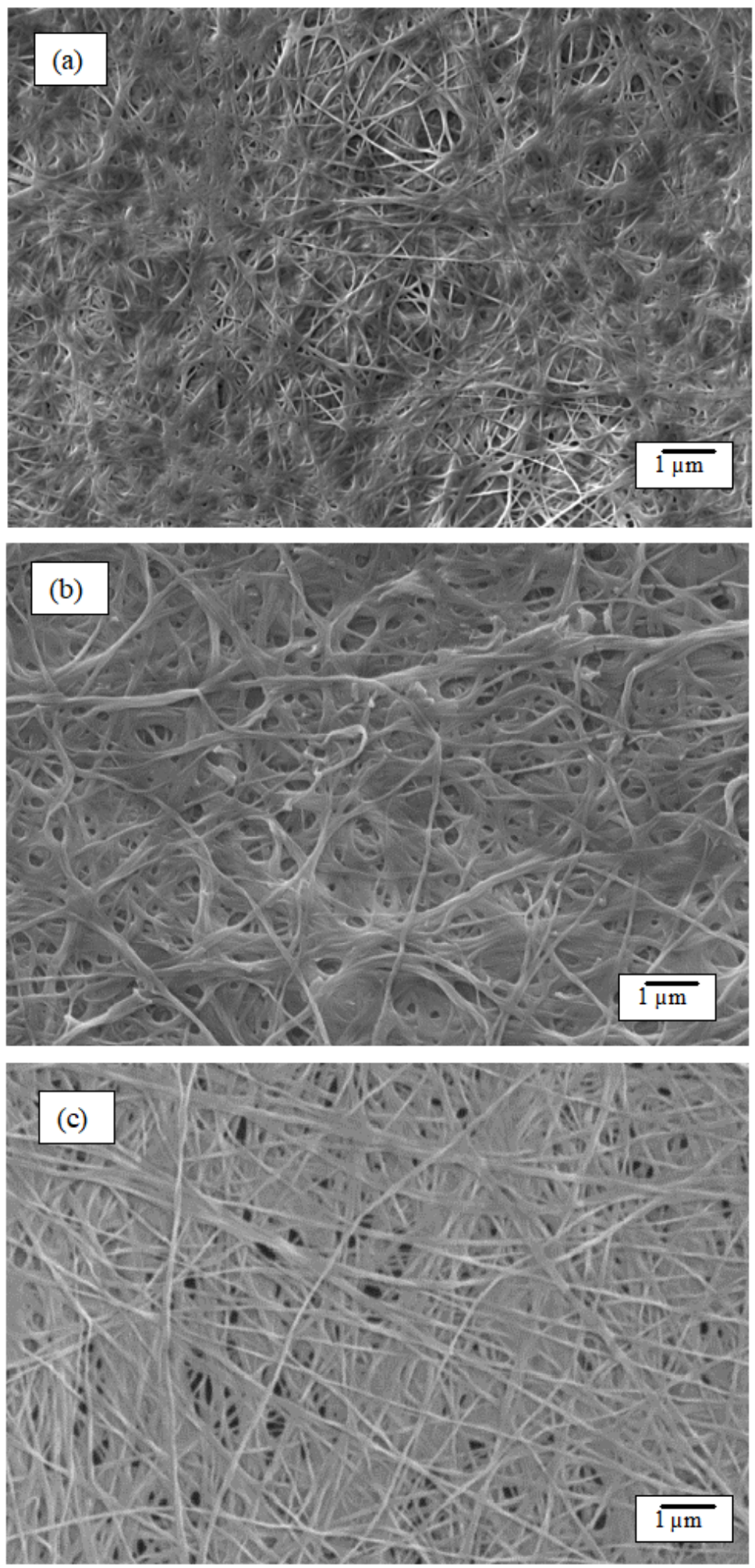

Figure 2

SEM images of a) $B C, b$ ) Agar-BC and c) Chitosan-BC 


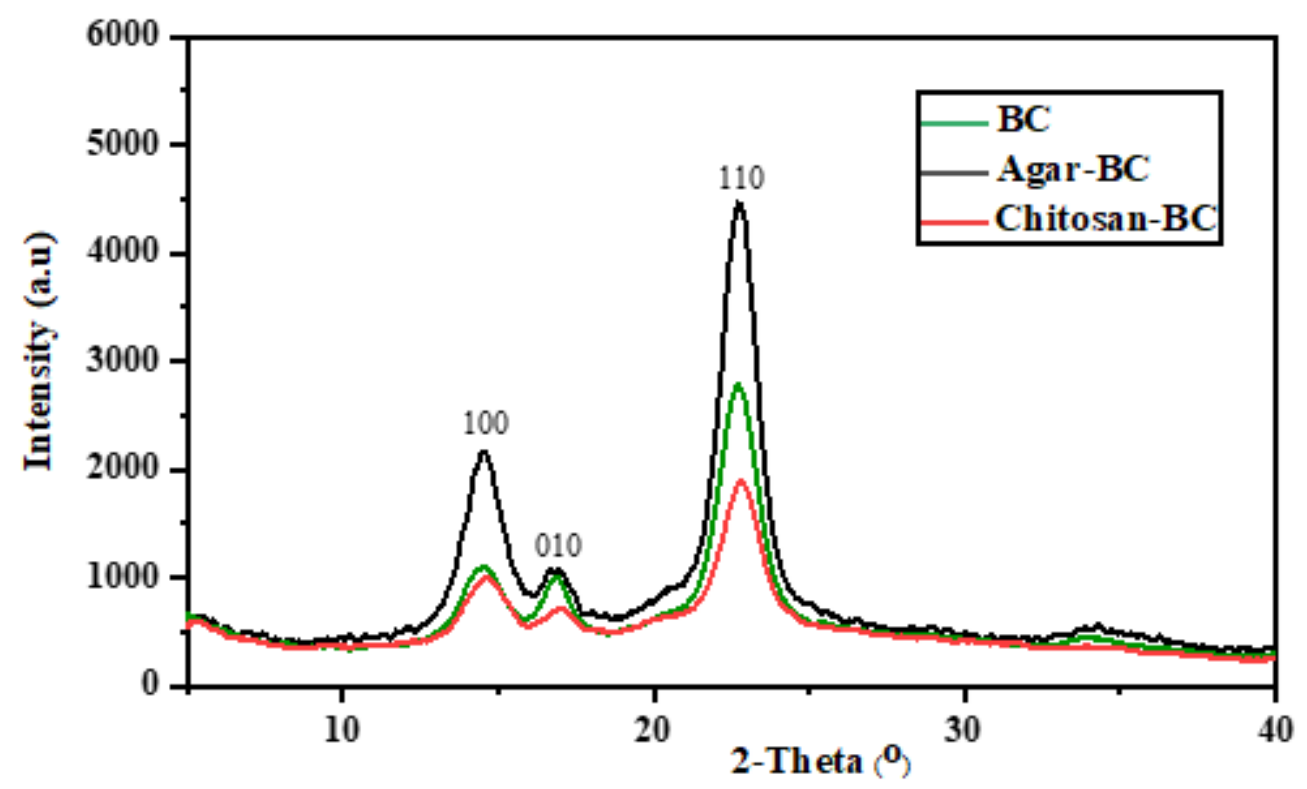

Figure 3

X-ray diffraction profiles of $\mathrm{BC}, \mathrm{Agar}-\mathrm{BC}$ and Chitosan-BC 

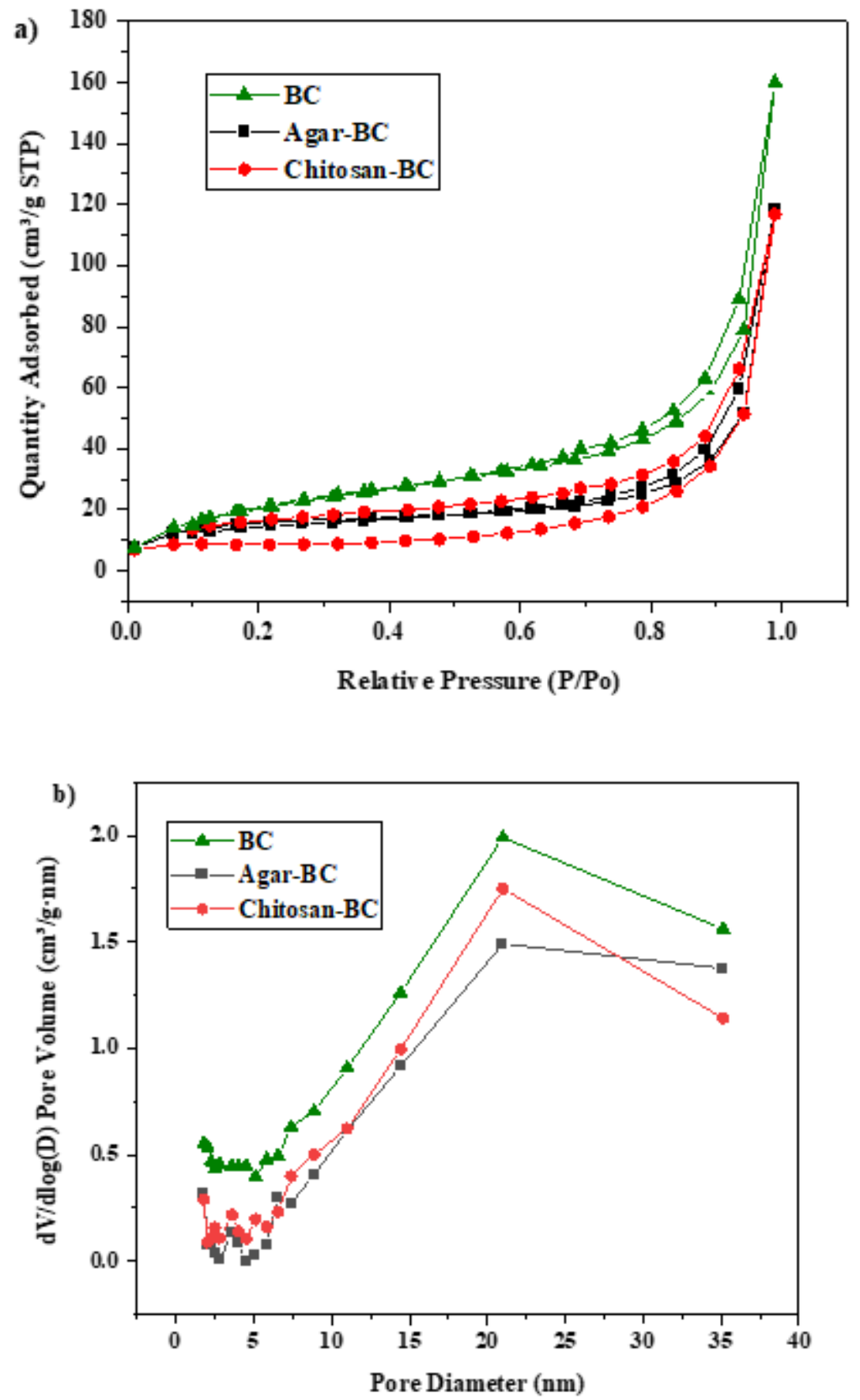

Figure 4

(a) Nitrogen adsorption isotherms and (b) Cumulative pore volume versus pore size of BC, Agar-BC and Chitosan-BC 

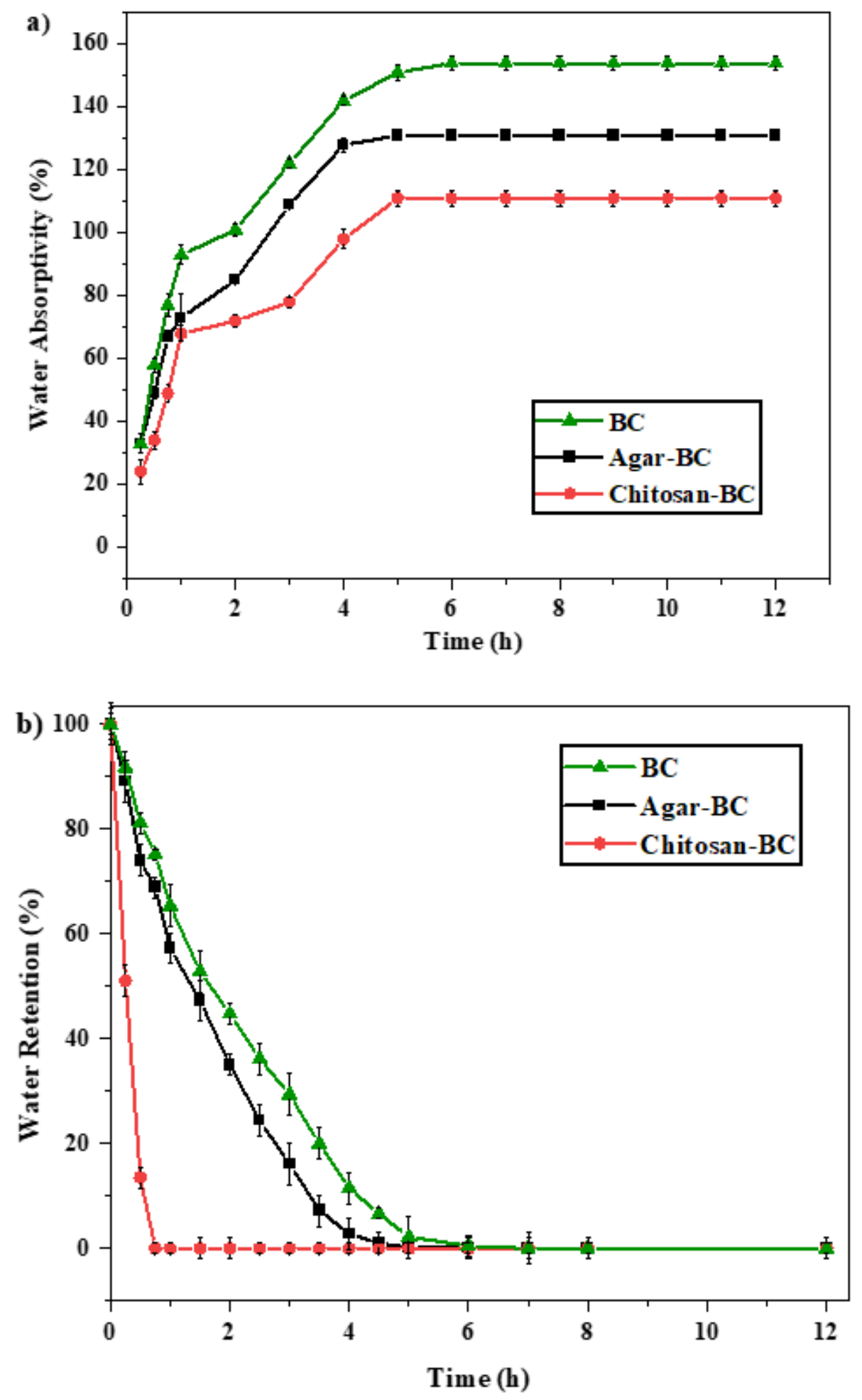

Figure 5

a) Water absorption and b) Water retention kinetics of $B C$, Agar-BC and Chitosan-BC 


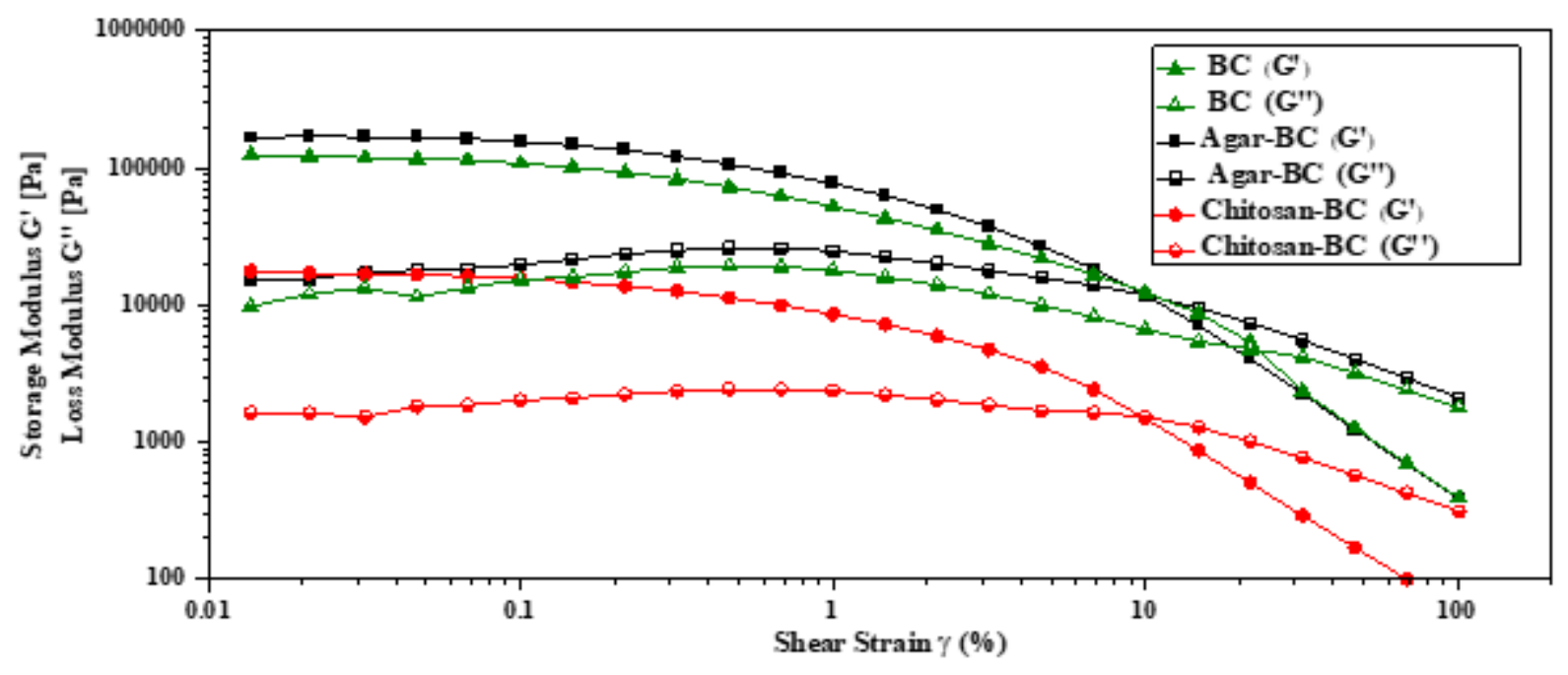

\section{Figure 6}

Variation in Storage modulus G' and loss modulus G" versus shear strain $\mathrm{Y}(\%)$ for $\mathrm{BC}$, Agar-BC and Chitosan-BC. 

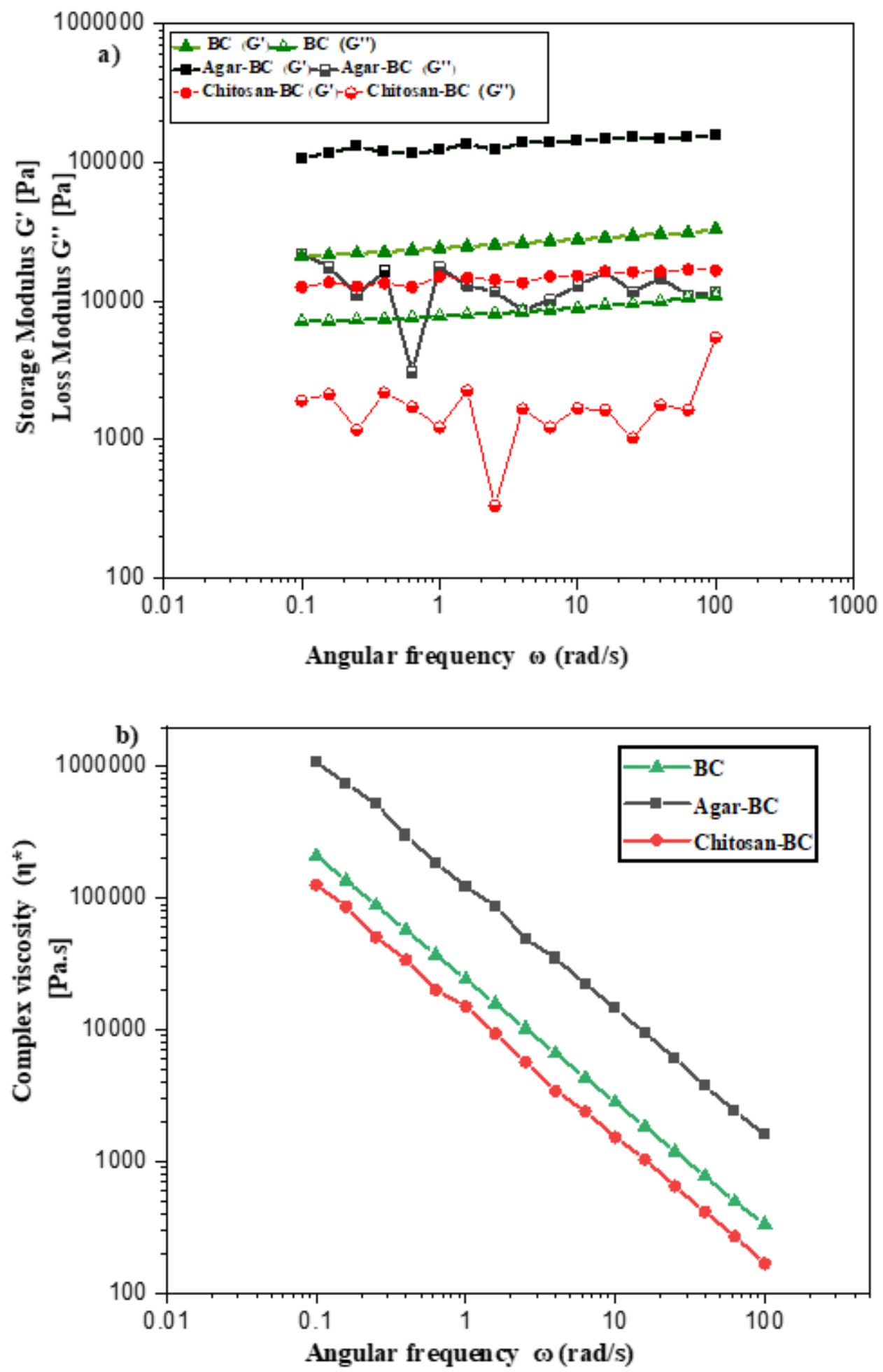

Figure 7

a) Variation in storage modulus $G^{\prime}$ and loss modulus G" versus angular frequency $\omega(\mathrm{rad} / \mathrm{s})$ and b) Variation in complex viscosity $\left|\eta^{\star}\right|$ versus the angular frequency $\omega(\mathrm{rad} / \mathrm{s})$ of $\mathrm{BC}$, Agar-BC and ChitosanBC. 

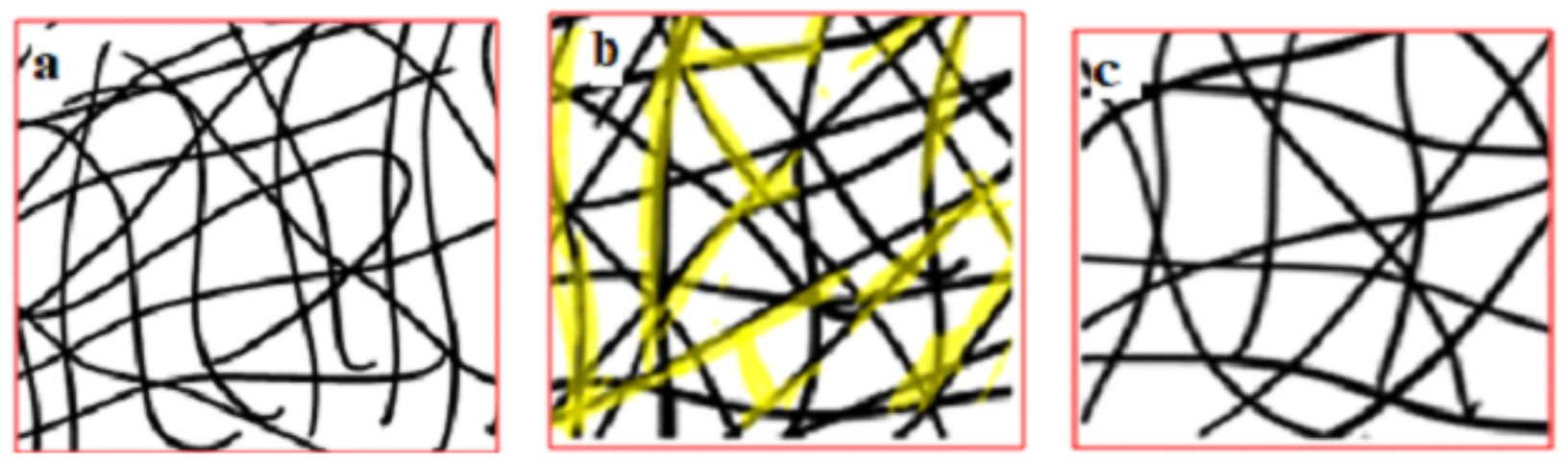

Figure 8

Schematic illustrations of the presumed fiber morphologies of a) BC, b) Agar-BC and c) Chitosan-BC 